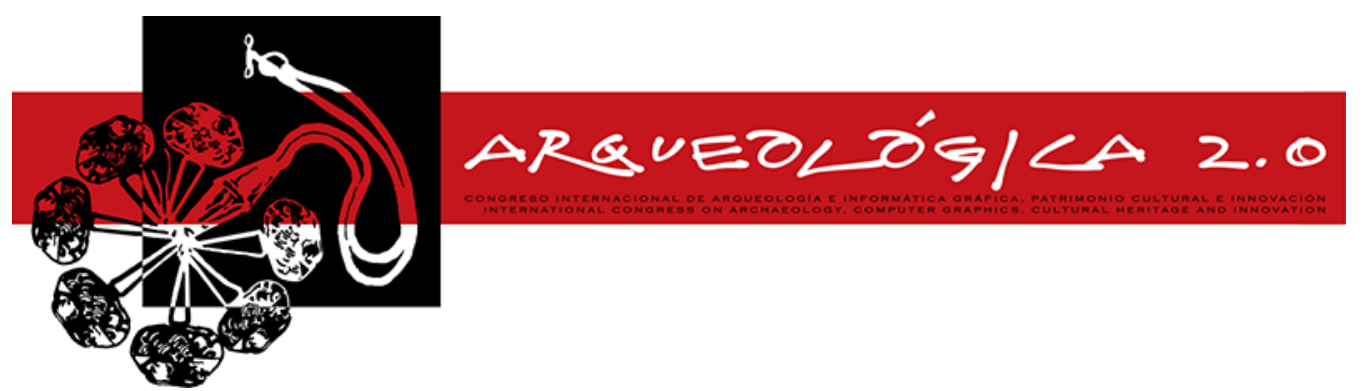

Proceedings of the $8^{\text {th }}$ International Congress on Archaeology,

Computer Graphics,

Cultural Heritage and Innovation

'ARQUEOLÓGICA 2.0'

in Valencia (Spain),

Sept. 5-7, 2016

DOI: http://dx.doi.org/10.4995/arqueologica8.2016.3510

\title{
PARAMETERIZATION OF COMPLEX CULTURAL HERITAGE SHAPES FOR ONLINE VIEWING AND INTERACTIVE PRESENTATION AND PROCESSING
}

\author{
Milan Ćurković*, Damir Vučina \\ FESB, Faculty of Electrical Engineering, Mechanical Engineering and Naval Architecture, University of Split, R. Boskovica 32, 21000 \\ Split, Croatia. milan.curkovic@fesb.hr; damir.vucina@fesb.hr
}

\begin{abstract}
:
We have developed algorithms and programs capable of efficiently parameterizing complex cultural heritage shapes including texture, which significantly reduces the data-set size. This is potentially significant for online viewing and interactive presentation and processing. The proposed approach is based on Non-uniform rational B-splines (NURBS) mathematical model which is also by itself suitable for analysis, especially of different artistic techniques.
\end{abstract}

Key words: virtual archaeology, digital archaeology, cultural heritage, documentation, 3D reconstruction, NURBS parameterization

\section{Introduction}

In recent times the cultural heritage worldwide becomes one of the main subjects in the field of information and communications technology (ICT). That way, cultural heritage is becoming available to all enthusiasts of culture and history for reviewing, learning and analysing, and all that over the web. The Virtual Museum (VM) will be a place for the critical personal preview before a possible visit to the museum and a place where people can renew their memories. But, implementing a virtual museum with all expected possibilities is a very demanding task. The main problem is to get the shape of cultural heritage objects in the form which does not take a lot of memory space and which provides real-time data transmission adequacy. That form should also be suitable for display and for shape analysis. This is not easy by using standard systems because the records of geometry and textures are too large for easy redistribution and dynamic display, and any analysis of them is almost impossible, especially in real-time.

We present enhanced single-patch NURBS parameterization based on fitting parameter values as a mathematical model which may also be considered as an approximation or as an initial solution for piecewise NURBS and T- spline parameterization (RiffnallerSchiefer et al. 2016; Dimitri et al. 2014). It relies on papers (Ćurković and Vučina 2014; Vučina et al. 2014) which outline a parameterization based on fitting parameter values and classification of 3D shape deviation using feature recognition operating on parameterization control points. The proposed approach should be considered as an interactive and adaptive compression of the shape and texture with the aim of visualization in real-time and also efficient analysis. The initial form of the 3D objects is a 3D point cloud obtained by a high resolution digitizing system, and the texture is a set of images.

The advantages of such a form is a very small number of parameters needed to display the shape. That way the transfer and archiving of such data becomes trivial.

In addition, the mere analysis of 3D objects using such a form is much easier because of the possibility of applying different mathematical functions (filters) in order to get the character of the shape.

\section{Data acquisition}

The equipment we have used in this paper is the ATOS system which consists of a projector and two cameras (GOM GmbH, 2012), that include stereophotogrammetry and triangulation (Peng and Gupta, 2007; Cyganek and Siebert, 2009), and which provides for efficient 3D scanning with high resolution and accuracy (Fig. 1). It is an optical system based on triangulation and stereo-photogrammetry which applies structured light patterns projection and uses time-based coding for addressing the positions of individual points. High resolution and high accuracy 3D optical scanning results in high density point clouds, typically in the range beyond 108 points. At present there is no ICT which is capable of providing efficient functionality based on such

*Corresponding Author: Milan Ćurković, milan.curkovic@fesb.hr 
initial 3D scanning results. That is the reason why we have started the development of a parametric model which adequate describes cultural heritage objects. In the following, we provide examples that represent a spectrum of heritage objects and demonstrate the robustness of our approach. The first sample shows two heads from the Cathedral of St. James in Sibenik (Fig. 1 ), Croatia and the second one represents the missing stone block from wall of Diocletian's Palace in Split, Croatia (Fig. 2).

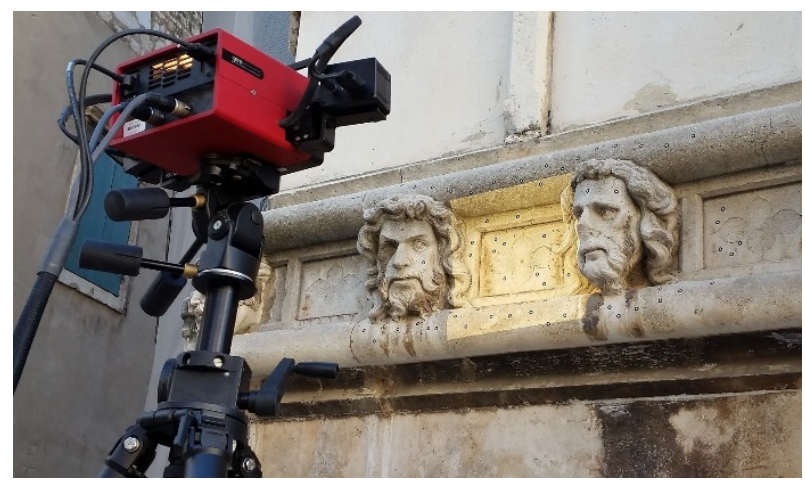

Figure 1: The scanning process of two heads from the Cathedral of St. James in Sibenik, Croatia.

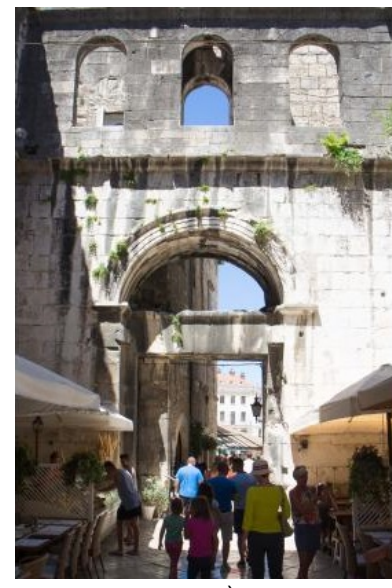

a)

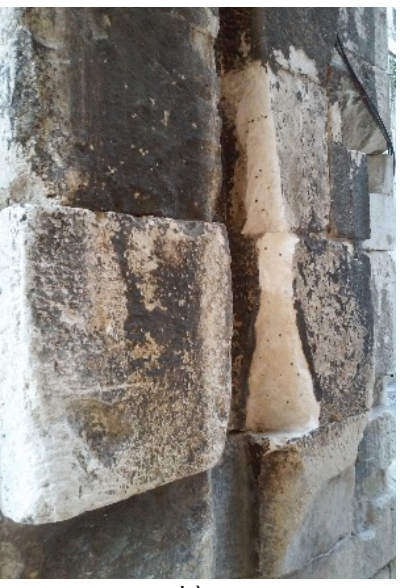

b)

$$
\boldsymbol{C}(u, v)=\frac{\sum_{i_{0}=0}^{n_{0}} \sum_{i_{1}=0}^{n_{1}} N_{i_{0}, p_{u}}(u) N_{i_{1}, p_{v}}(v) w_{i_{0} i_{1}} \boldsymbol{Q}_{i_{0} i_{1}}}{\sum_{i_{0}=0}^{n_{0}} \sum_{\substack{i_{1}=0 \\ n_{1}}} N_{i_{0}, p_{u}}(u) N_{i_{1}, p_{v}}(v) w_{i_{0} i_{1}}}
$$

where $\mathrm{n}_{0}, \mathrm{n}_{1}$ are the numbers of the control points, $\mathrm{N}_{\mathrm{i}_{0}, \mathrm{p}_{\mathrm{u}}}(\mathrm{u})$ and $\mathrm{N}_{\mathrm{i}_{1}, \mathrm{p}_{\mathrm{v}}}(\mathrm{v})$ are the basic B-spline functions of degrees $\mathrm{p}_{\mathrm{u}}, \mathrm{p}_{\mathrm{v}} \in \mathbb{N}$ defined by

$$
\begin{gathered}
N_{i, 0}(u)=\left\{\begin{array}{l}
1, \bar{u}_{i} \leq u \leq \bar{u}_{i+1}, \\
0, \text { else }
\end{array}\right. \\
N_{i, p}(u)=\frac{u-\bar{u}_{i}}{\bar{u}_{i+p}-\bar{u}_{i}} N_{i, p-1}(u)+\frac{\bar{u}_{i+p+1}-u}{\bar{u}_{i+p+1}-\bar{u}_{i+1}} N_{i+1, p-1}(u) .
\end{gathered}
$$

The knots $\bar{u}_{i} \in[0,1]$ as part of the basic B-spline functions we set as

$$
\overline{\boldsymbol{u}}=\{\underbrace{0, \ldots 00}_{p+1}, \bar{u}_{i+p}, \ldots, \bar{u}_{n-p-1}, \underbrace{1, \ldots 1}_{p+1}\},\left\{\bar{u}_{i}=\frac{i}{n}\right\}_{i=p+1}^{n-p-1},
$$

and they are fixed during the parameterization process. The matrix

$$
\boldsymbol{W}=\left[\begin{array}{ccc}
w_{00} & \cdots & w_{0 n_{1}} \\
\vdots & \ddots & \vdots \\
w_{n_{0} 0} & \cdots & w_{n_{0} n_{1}}
\end{array}\right]
$$

denotes the weight factors which we all set as 1 for the initial solution.

To fit the NURBS model to a given shape, the proposed approach uses the section matrix $P \in \mathbb{R}^{3\left(m_{0}+1\right) \times\left(m_{1}+1\right)}$ from the shape by applying parallel sections instead of using the original unstructured shape. During the fitting procedure, each point $\mathrm{P}_{\mathrm{j}_{0} \mathrm{j}_{1}}$ is assigned a pair of parameter values $\mathrm{u}_{\mathrm{i}_{0} \mathrm{j}_{1}}$ and $\mathrm{v}_{\mathrm{j}_{0} \mathrm{j}_{1}} \in\left[\begin{array}{ll}0 & 1\end{array}\right]$ given according to

$$
\left\{u_{j_{0} 0}=u_{j_{0} 1}=\cdots=u_{j_{0} m_{1}}:=\frac{j_{0}}{m_{0}}\right\}_{j_{0}=0}^{m_{0}}
$$

and

Figure 2: Diocletian's Palace in Split, Croatia: a) The Iron Gate (http://www.visitsplit.com/en/523/the-iron-gate); b) The missing stone block from wall.

\section{NURBS model construction}

The aim of construction of a NURBS surface is to get a small (typically in range less than 100 kilobytes) parameter data set which faithfully describes the object shape instead of the initial high density 3D scanned point cloud (often measured in gigabytes). In the following examples (the small points in Figure 4 and Figure 8) the main part of the NURBS model is shown, the set of the control points. The additional part of the NURBS surface, the set of weighted parameters used for the additional quality, is hidden from the users.

The NURBS surface is defined by its control points, weight factors, degrees of polynomials and the set of knots,

$$
\left\{v_{0 j_{1}}=v_{1 j_{1}}=\cdots=v_{m_{0} j_{1}}:=\frac{j_{1}}{m_{1}}\right\}_{j_{1}=0}^{m_{1}} .
$$

The NURBS surface which represents the geometry of the sections matrix $P$ must minimize the error function

$$
\begin{aligned}
& \boldsymbol{E}(\boldsymbol{U}, \boldsymbol{V}, \boldsymbol{Q}, \boldsymbol{W})= \\
& =\frac{1}{2} \sum_{j_{0}=0}^{m_{0}} \sum_{j_{1}=0}^{m_{1}}\left\|\boldsymbol{C}\left(u_{j_{0} j_{1}}, v_{j_{0} j_{1}}\right)-\boldsymbol{P}_{j_{0} j_{1}}\right\|^{2}
\end{aligned}
$$

which constitutes a least-squares problem. Minimizing the error function (5) reduces the cumulative sum of distances between the geometry $\mathrm{P}_{\mathrm{j}_{0} \mathrm{j}_{1}}$ and the corresponding points on the NURBS surface

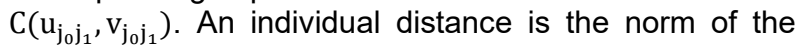
individual error vector.

The result of this numerical method is the desired set of the control points and their associated weight factors which together form the small input data for the Virtual Museum. Since the proposed method is non-linear, as 
the initial solution it uses the solution of linear best-fitting of B-spline surfaces to data-sets acquired by measurement and numerically generated surfaces (Eberly, 2010). It is realistic to expect that the users of the virtual museum, while previewing some object would like to zoom in and see specific details. To make this possible, it is necessary to construct one detailed parametric model with a larger number of parameters or one simple model with an additional series of small simple parametric models that individually describe only certain parts of the object. While zooming, these are being superimposed on the initial simple parametric model.

The above example (Fig. 3) presents one of the results of the 3D scanning process of the two-head set from the Cathedral of St. James in Sibenik, Croatia, as a standard example from the cultural heritage. The original 3D scanned size is about $1.5 \mathrm{~GB}$ and its thinned (CAD) version is about $100 \mathrm{MB}$.

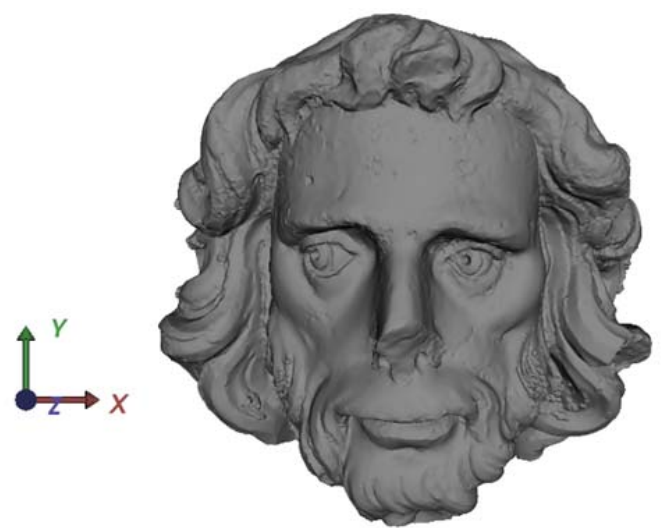

Figure 3: One of the results of the 3D scanning process of the two head set from the Cathedral of St. James in Sibenik, Croatia.

Although its thinned version is reduced by 15 -times, it is still too big for fluid manipulation. The next sample (Fig. 4) presents the proposed NURBS model of the above example. The model consists of a $40 \times 40$ control points grid and $40 \times 40$ weight factors grid which take up $54 \mathrm{~KB}$ of memory.

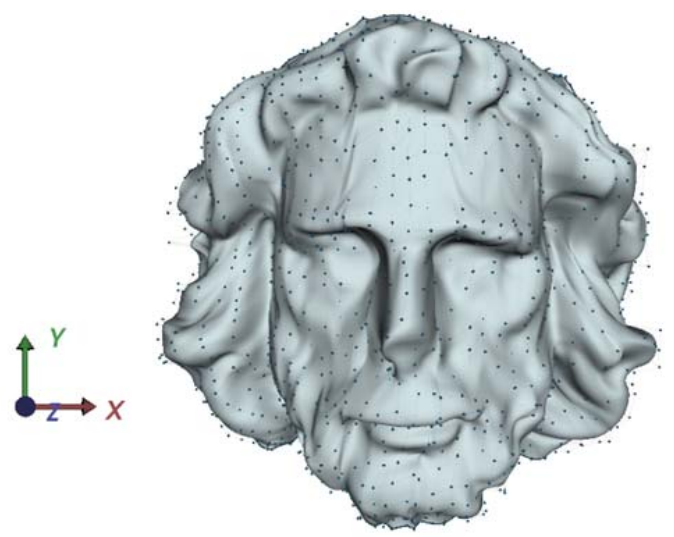

Figure 4: The single-patch NURBS surface as a result of the parameterization process, $40 \times 40$ control points, the degree of B-spline functions is 2 .
The distribution of the geometric error between the original scanned surface (Fig. 3) and the NURBS model (Fig. 4) is shown in Figure 5.

The geometric error is relevant for possibly improved fitting by the proposed method or towards selection of some other methods that are based on the proposed method for further fitting. The criterion could be the cumulative geometric error or it could be the error obtained by using mathematical filters in areas with geometric features (peaks, edges, ...).

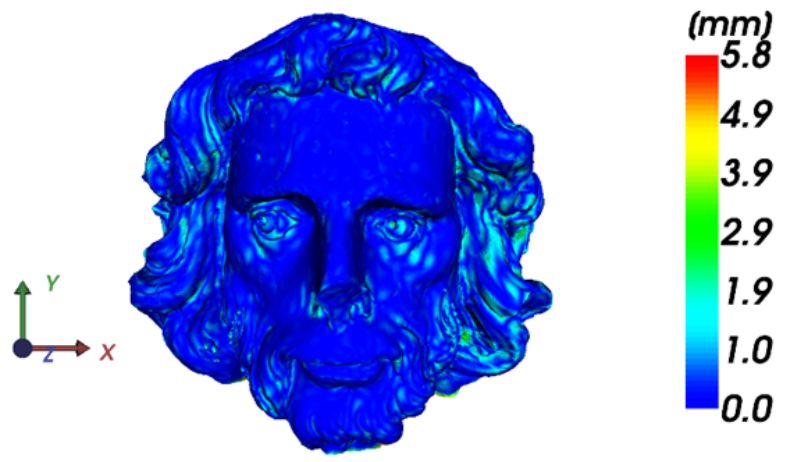

Figure 5: The distribution of the geometric error between original scanned surface (Fig. 3) and NURBS model (Fig. 4).

As preparation for the proposed method, we have used the projection of the initial object's shape into a rectangular domain and that way we are able to employ the proposed method on non-rectangular patches (Fig. 6 ). This is very important in terms of multi-patch NURBS parameterization because it is not necessary to have a rectangular subdivision of shape, and it is posible to have nature borders of the patches.

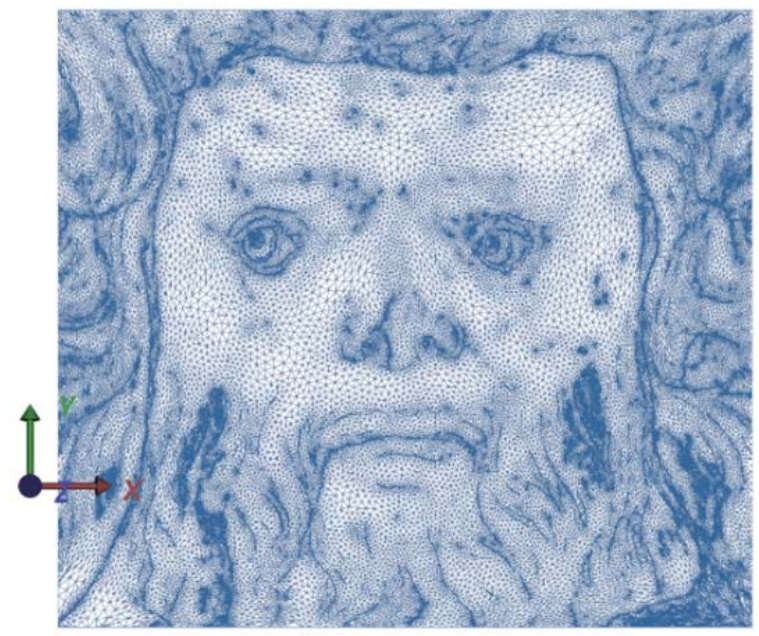

Figure 6: Projection into rectangular domain of thinned surface (Fig. 3).

The other preparation method we have applied is remeshing of the original shape in order to achieve higher density of the shape points in areas with significant geometric features. The method results in a better global fitting solution and more accuracy in the mentioned areas. We detect areas with significant geometric features by applying the Principal component analysis (PCA) method, where the ratio of eigen-values identifies the geometric feature. For a local sub-set of the point cloud, the variance measures the spread of 
data along a single dimension in the data set, and the covariance measures the mutual dependence of any two dimensions of the data-set

$$
\operatorname{var}(x)=\frac{\sum_{i=1}^{n}\left(x_{i}-\bar{x}\right)^{2}}{n-1}
$$

and

$$
\operatorname{cov}(x)=\frac{\sum_{i=1}^{n}\left(x_{i}-\bar{x}\right)\left(y_{i}-\bar{y}\right)}{n-1}
$$

For example, while positive covariance in a local point cloud sub-set indicates the two dimensions which increase or decrease together, low covariance implies mutually independent dimensions in the sub-set. Principal component analysis can be very useful with this regard as it may assist in identifying features contained in the data set by transforming correlated variables into new variables with less correlation. Accordingly, principal component analysis can identify the variables responsible for the majority of variation in the local point cloud sub-set.

The proposed approach we also applied on the missing stone block from wall from the Diocletian's Palace in Split (Fig. 7). The original 3D scanned size is about 2.9 $\mathrm{GB}$ and its thinned (CAD) version is about $160 \mathrm{MB}$.

The missing stone block is an excellent example for applying of a mathematical model such as the proposed NURBS model. In addition to the reduction of the number of parameters needed to describe the geometry and facilitated reviewing within the Virtual Museum, it represents a classic example of a damaged object which should be numerically analyzed and faithfully replicated.

These mentioned numerical procedures are significantly facilitated by the existence of a parametric model. This is because the topological structure is included by itself in the parametric model which enables the application of integral filters.

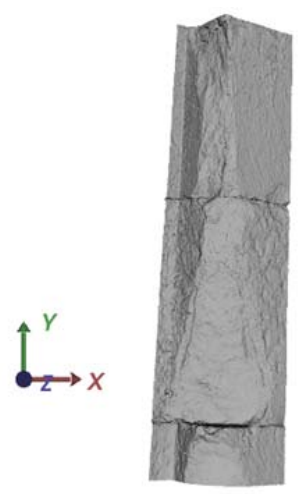

a)

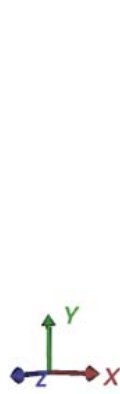

b)

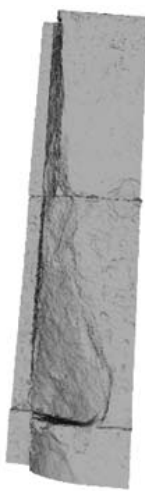

Figure 7: Two views of the result of the 3D scanning process of the wall from the Diocletian's Palace in Split.

The next sample (Fig. 8) presents the proposed NURBS model of the sample (Fig. 7). The model consists of a $20 \times 20$ control points grid and a $40 \times 20$ weight factors grid which take up $14 \mathrm{~KB}$ of memory.

The distribution of the geometric error between the original scanned surface (Fig. 7) and the NURBS model
(Fig. 8) is shown in Figure 9. The geometric error in this example has a micro character which confirms the quality of the initial parametric model. Further improvements related to the texture that can be part of a separate parametric model.

In terms of replacing the missing parts of a cultural heritage object, the geometric difference (texture) shown in Figure 9 should be minimized as much as possible since the process of replacing the missing part should include the texture from the surrounding area to substitute the original texture. To achieve that requirement the proposed parametric model should be capable to present texture also. That is possible with an additional parameterization model which will be superimposed on the initial parametric model (Fig. 8). Converting texture into a separate parametric model and superimposing it onto the basic parameterization model is generally an extensive and demanding topic and hence a subject of future work, only outlined in this paper.

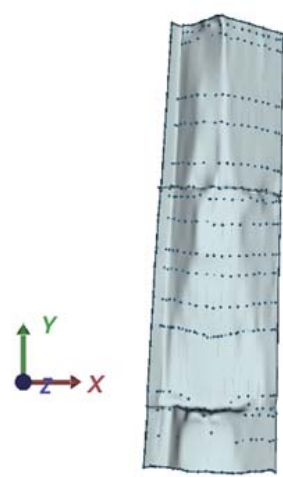

a)

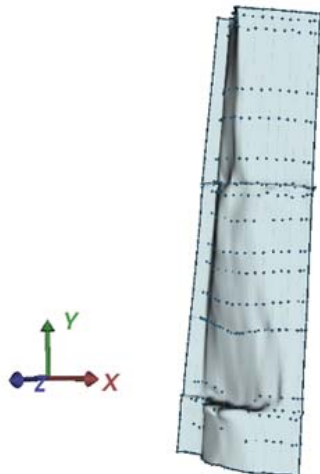

b)
Figure 8: Two views of the single-patch NURBS surface as a result of the parameterization process, $20 \times 20$ control points, the degree of B-spline functions is 2 .

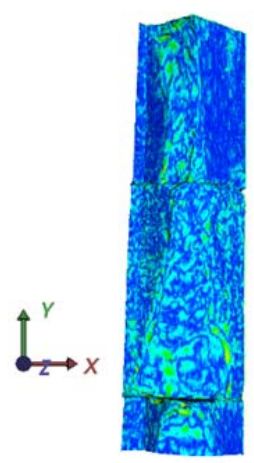

a)
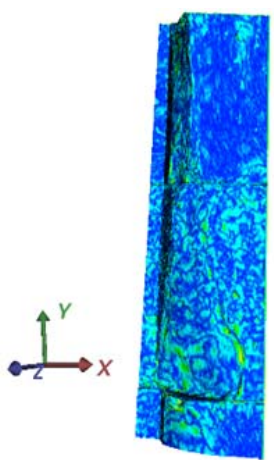

b)

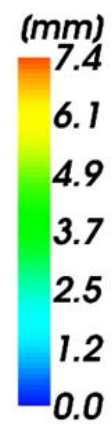

6.1
Figure 9: Two views of the distribution of the geometric error between original scanned surface (Fig. 7) and NURBS model (Fig. 8).

\section{Conclusion}

As a dynamic preparation method, the proposed approach goes hand in hand with tools such as the 3D HOP (http://3dhop.net/). The shape of the object is presented as two superimposed models. The basic NURBS model applies to the global geometry and the other model to texture. During previewing by means of a Virtual Museum based on tools such as 3D HOP, it could 
be converted to one of the supported data formats (ply, ...). All the necessary steps for the conversion could be performed on the client side in real time.

\section{Acknowledgements}

This work was supported by the Croatian Science Foundation [grant number IP-2014-09-6130]

\section{References}

ĆURKOVIĆ, M. and VUČINA, D., 2014. 3D shape acquisition and integral compact representation using optical scanning and enhanced shape parameterization, Advanced Engineering Informatics, 28(2), pp. 111-126.

DIMITRI, R., DE LORENZIS, L., SCOTT, M.A., WRIGGERS, P., TAYLOR, R.L. and ZAVARISE, G., 2014. Isogeometric large deformation frictionless contact using T-splines, Comput. Methods Appl. Mech. Engrg. 269, pp. 394-414.

DIMITRI, R., DE LORENZIS, L., WRIGGERS, P. and ZAVARISE, G., 2014. NURBS- and T-spline-based isogeometric cohesive zone modelingof interface debonding, Computational Mechanics, 54(2), pp. 369-388.

EBERLY, D., 2010. Least-Squares Fitting of Data with B-Spline Curves. Available: http://www.geometrictools.com [1/1, 2010].

GOM GmbH, 2012. Available: http://www.gom.com/metrology-systems/system-overview/atos.html [2/7, 2012].

LI, X. and SCOTT, M.A., 2013. Analysis-suitable T-splines: characterization, refineability, and approximation, submitted for publication, arXiv: 1211.5669 [CS.GR]

NGUYEN, V.P., KERFRIDEN, P., BRINO, M., BORDAS, S.P.A. and BONISOLI, E., 2013. Nitsche's method for two and three dimensional NURBS patch coupling, Computational Mechanics, 53(6), pp. 1163-1182.

PENG, T. and GUPTA, S.K., 2007. Model and algorithms for point cloud construction using digital projection patterns, ASME Journal of Computing and Information Science in Engineering, 7(4), pp. 372-381.

VUČINA, D., ĆURKOVIĆ, M. and NOVKOVIĆ, T., 2014. Classification of 3D shape deviation using feature recognition operating on parametrization control points, Computers in industry, 65(6), pp. 1018-1031.

CYGANEK, B. and SIEBERT, J.P., 2009. An Introduction to 3D Computer Vision Techniques and Algorithms, John Wiley \& Sons, Chichester.

RIFFNALLER-SCHIEFER, A., AUGSDÖRF, U.H. and FELLNER D.W., 2016. Isogeometric shell analysis with NURBS compatible subdivision surfaces. Applied Mathematics and Computation, 272, pp. 139-147. 